\title{
Computerized axial tomography: the normal EMI scan
}

\author{
J. GAWLER, J. W. D. BULl, G. H. DU BOULAY, AND J. MARShALL \\ From the National Hospital for Nervous Diseases, Queen Square, London
}

SYNOPSIS Computerized axial tomography using the EMI Scanner s a new method of using $x$-rays in diagnosis. The technique displays intracranial and orbital structures in the transverse plane. The appearances of normal EMI Scans are described and correlated with cerebral and orbital anatomy seen in transverse section.

Computerized transverse axial tomography with the EMI Scanner-a revolutionary method of utilizing $x$-rays in diagnosis developed by $\mathrm{G}$. N. Hounsfield-provides images of transverse 'slices' of the brain without the use of contrast media. Normal structures, such as the internal capsule, which could not be shown by conventional techniques, and structures, such as the ventricular system, which could be seen only with the aid of contrast media, may now be displayed without prior disturbance.

The present paper describes the normal intracranial anatomy as revealed by the EMI Scan. As normal volunteers have not been examined, the composite picture described here has been built from experience with 2500 patients investigated for intracranial disease, in some of whom the EMI Scan and other investigations proved negative. Full descriptions of the scanning technique have been published elsewhere (Hounsfield, 1973) and only relevant features will be considered here.

Each transverse 'slice' of brain is considered as a matrix of cells. Initially, an 80 by 80 matrix containing 6400 cells each $3 \mathrm{~mm}$ by $3 \mathrm{~mm}$ was used, but currently a 160 by 160 matrix of 25600 cells measuring $1.5 \mathrm{~mm}$ by $1.5 \mathrm{~mm}$ is employed. The depth of each cell is 8 or $13 \mathrm{~mm}$ depending upon the adjustable width of the $x$-ray beam. The image of the brain is constructed from measurements of the amount of $x$-ray absorbed by each of the cells as the $x$-ray beam scans the head. The absorption values are expressed on an arbitrary scale where air has a

(Accepted 23 May 1975.)

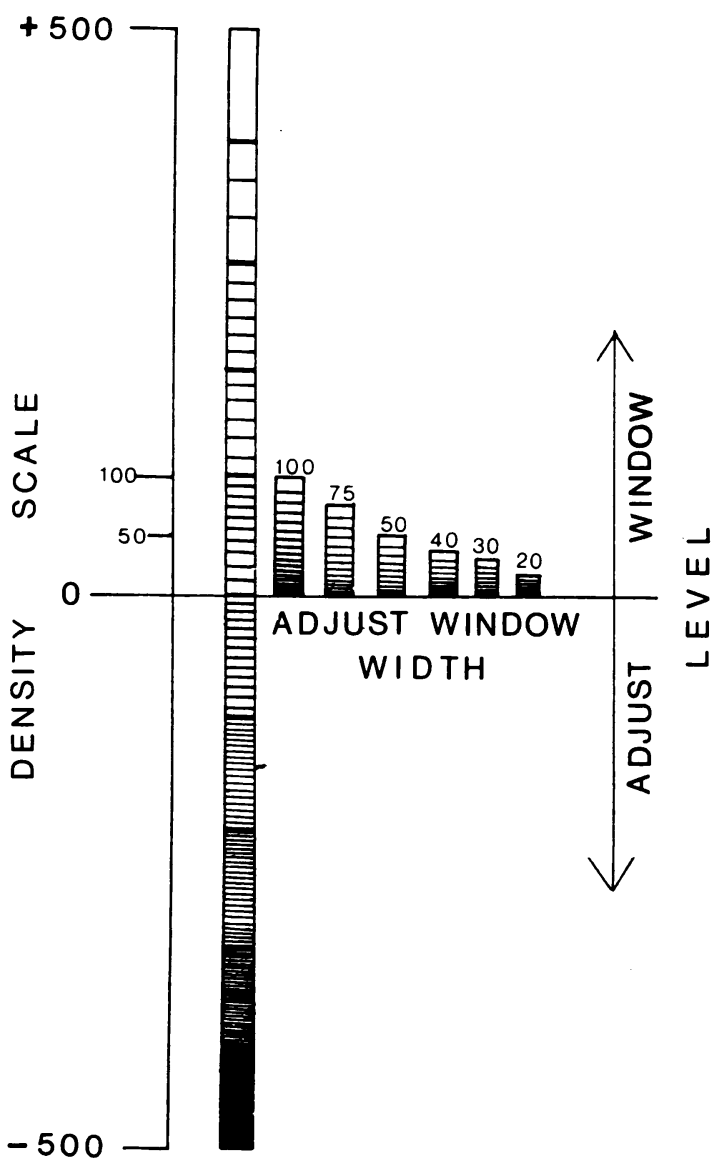

FIG. 1 Oscilloscope display represented graphically. The ten divisions (peak black, peak white and eight shades of grey) may be applied to the entire density range used by the EMI system or restricted to any 935 


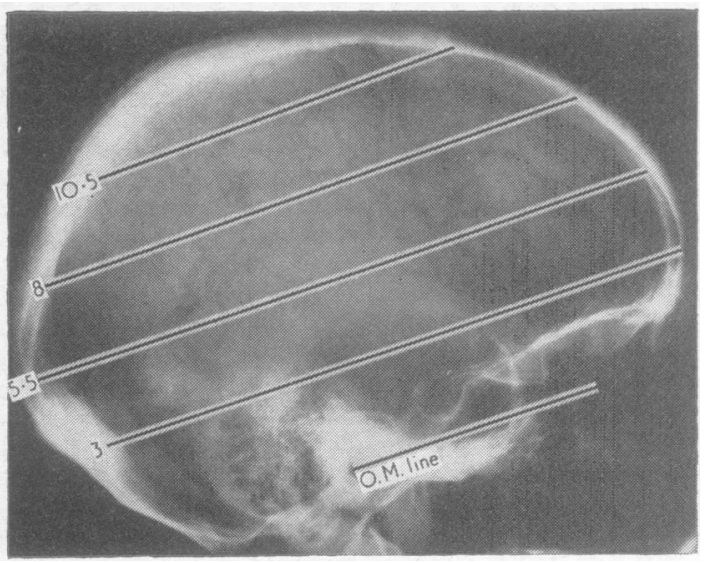

FIG. 2 Paired transverse axial tomograms, each 13 $\mathrm{mm}$ deep, are centred at regular intervals above the orbitomeatal line to cover the supratentorial compartment.

value of -500 , water has zero value, and dense bone a mean value of +500 . Cerebrospinal fluid gives values of 0 or 1 , white matter 10 to 18 , and grey matter 18 to 30 . Calcified structures such as the pineal body or choroid plexus give higher values.

The absorption values may be expressed numerically by a line printer or displayed as an image by a cathode ray oscilloscope whose adjustable grey scale has 10 divisions from peak

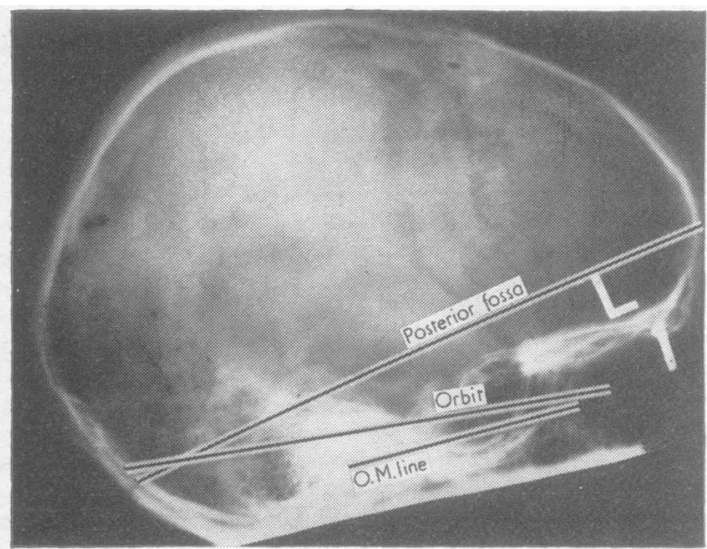

FIG. 3 Tomograms are centred specifically for posterior fossa and orbital examination. black (low density) to peak white (high density). The scale can be adjusted to cover the entire density range $(-500$ to +500$)$ or a segment from any part of the range (Fig. 1). Absorption values are calculated to an accuracy better than half per cent so that on the arbitrary scale a difference of 4 or 5 points is significant. When the oscilloscope scale covers 0 to 40 of the density range, each change in the grey scale represents 4 points of density difference permitting cerebrospinal fluid, grey and white matter to be distinguished.

For examination of supratentorial structures a series of four scans yielding eight $13 \mathrm{~mm}$ tomograms is employed. The tomogram pairs are centred $3,5.5,8$, and $10 \mathrm{~cm}$ above and parallel to the orbitomeatal line (Fig. 2). For posterior fossa examination the beam is centred 3 or $3.5 \mathrm{~cm}$ above the orbitomeatal line, with the patient's head flexed 15 or 20 degrees, and for the orbits a pair of $8 \mathrm{~mm}$ 'slices' centred 1 or $1.5 \mathrm{~cm}$ above the orbitomeatal line with the head extended 10 to 15 degrees are employed (Fig. 3).

\section{ANATOMY DEFINED BY THE EMI SCAN Intracranial compartment (Fig. 4)}

Because the size and shape of the skull varies from person to person a 'slice' at a given level above the orbitomeatal line will contain different structures in different patients. For this reason, a series of idealized 'slices' will not be described and, instead, the appearance and relationship of normal structures in the transverse plane as revealed by computerized tomography will be considered.

VENTRICULAR SYSTEM The lateral ventricles are seen in three or four contiguous 'slices' (Fig. 5). Considered from above downwards the highest 'slice' may incorporate only 1 or $2 \mathrm{~mm}$ of each lateral ventricle, which then appear as bilateral vague areas of slightly lower density (Fig. 6a). With slight obliquity of the head, one ventricle may just present within the 'slice', while the other lies below it. This can lead to the mistaken interpretation that a pathological diminution of white matter density exists in the centrum semiovale on this side (Fig. 6b). It must be remembered that as a 'slice' is $13 \mathrm{~mm}$ deep it may incorporate both cerebrospinal fluid and brain tissue with absorption values varying between 1 and 10 (Fig. 7). The precise limits of the ventricles cannot therefore be defined. In practice, this is not a 

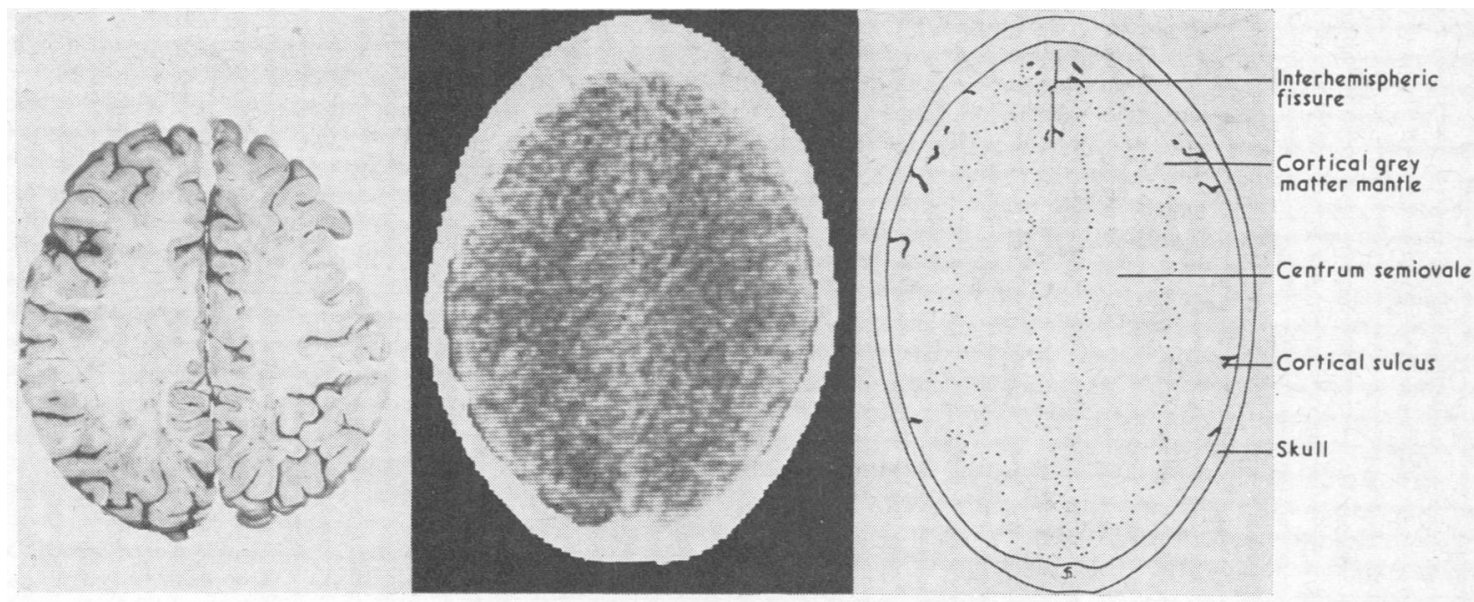

FIG. 4/1
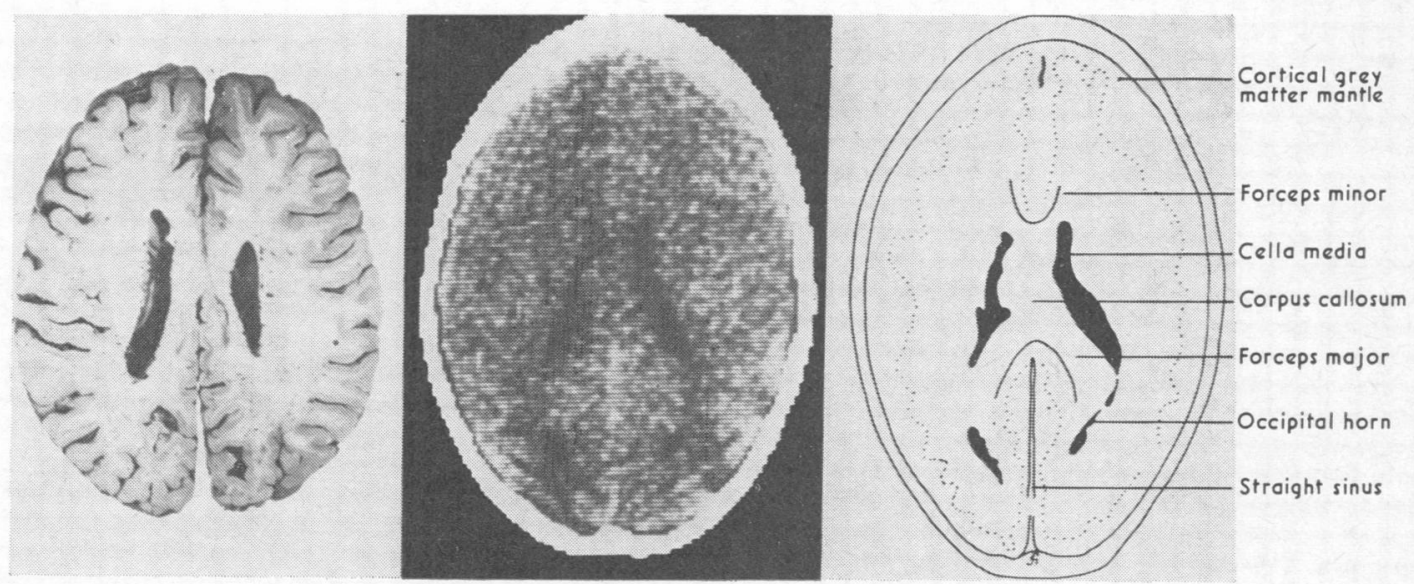

FIG. 4/2

FIG. 4 Normal EMI Scan. Three scan pairs centred 3 (4/5 and 6), 5.5 (4/3 and 4), and 8 (4/1 and 2) cm above the orbitomeatal line provide a sequence of six contiguous tomograms. A normal brain (left) has been cut at appropriate levels to match the scan and the diagram (right) shows those structures which may be identified on the scan. When comparing the cut surface of the brain and EMI Scan, it is important to remember the latter represents all the tissues in a 'slice' $13 \mathrm{~mm}$ thick. (See over.)

problem because, when the absorption values are contrasted on the oscilloscope, fairly sharp definition of the ventricles is obtained. When the ventricular margin lies within cells that contain principally cerebral tissues, a significant reduction in density may not be apparent and the ventricles would thus appear smaller than their true dimensions. Conversely, when a cell contains sufficient fluid to reduce its density, the whole cell is registered as ventricle, even though only a segment of the ventricle is projected into the cell, and this would make the ventricle appear larger than its real size. Overall, the technique makes the ventricles appear slightly smaller than in a fixed specimen of a normal brain, and this is allowed for in the interpretation.

The highest tomogram to pass clearly through the 

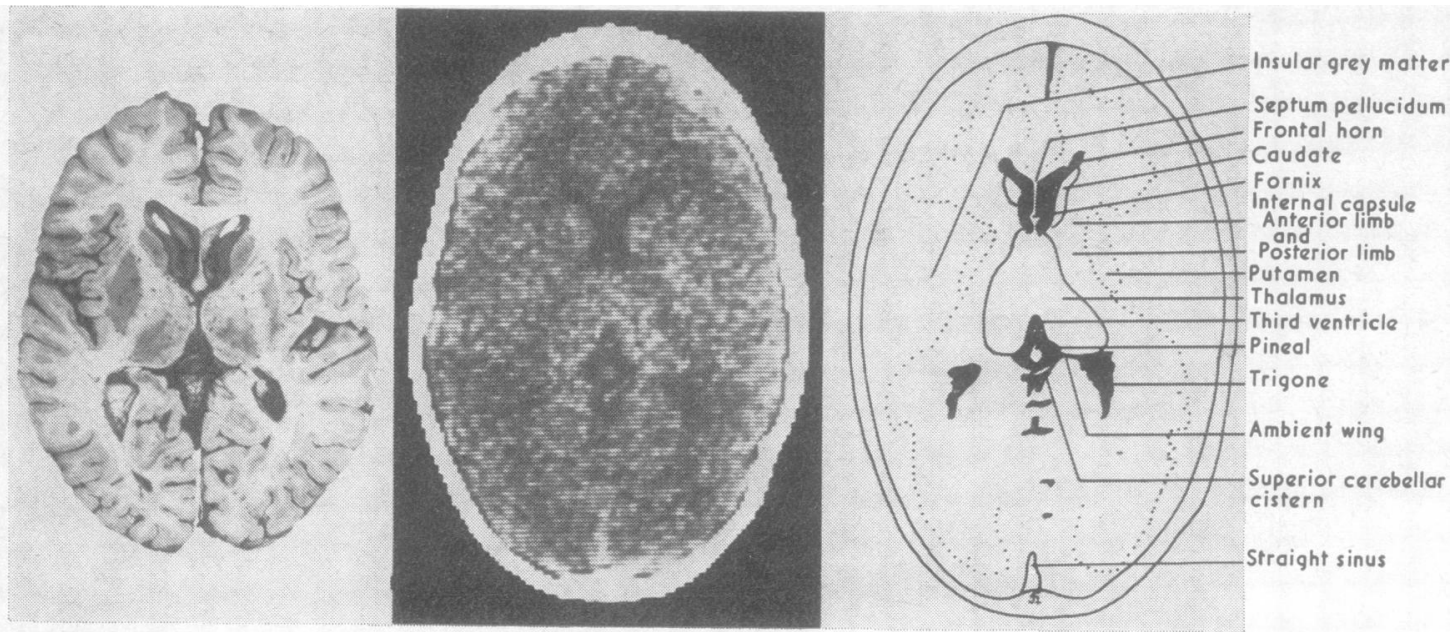

FIG. $4 / 3$
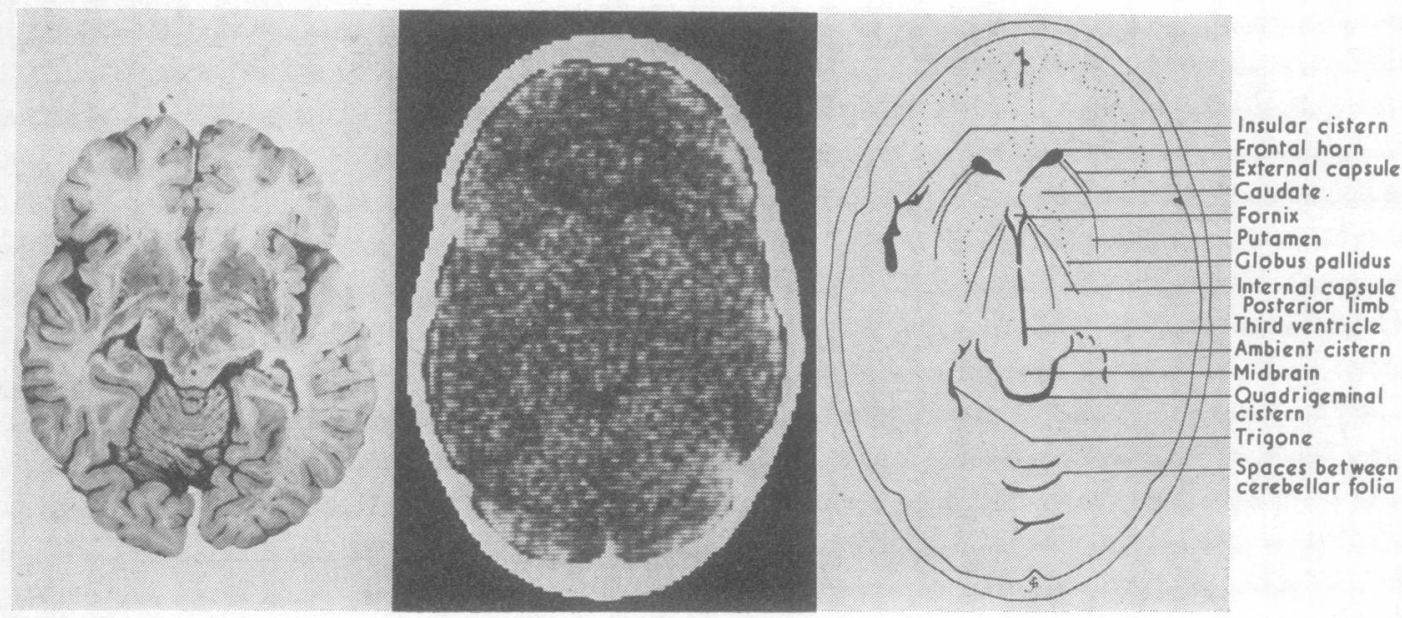

FIG. $4 / 4$

lateral ventricles contains the superior segments of the cellae mediae (Fig. 4/2). While these are fairly symmetrically displayed with respect to the midline, they commonly show some difference in size and contour. The body of the corpus callosum lies between the cellae mediae on this plane, with the forceps minor diverging anteriorly and the forceps major posteriorly along the medial aspects of the ventricles. Laterally lies the white matter of the corona radiata. The superior segments of the trigones and occipital horns may also be incorporated into this tomogram. Marked asymmetry of the occipital horns is common and not infrequently one or both contain insufficient cerebrospinal fluid for their clear delineation on the scan. A thin occipital horn may appear as a vague area of low density extending backward towards the occipital pole and should not be mistaken for a lesion. The next contiguous tomogram, descending through the series, incorporates the frontal horns anteriorly and the trigones and occipital horns posteriorly (Fig. 4/3). A degree of asymmetry in lateral ventricular contour is common, partly because of true anatomical difference and partly because any obliquity of the head will result in slightly different ventricular segments being incorporated on the two sides. Between the frontal horns, the septum pellucidum can be regularly identified on the 160 by 160 matrix but not always on the 80 by 80 matrix. At the posterior end of the septum the anterior columns of the fornix can be 

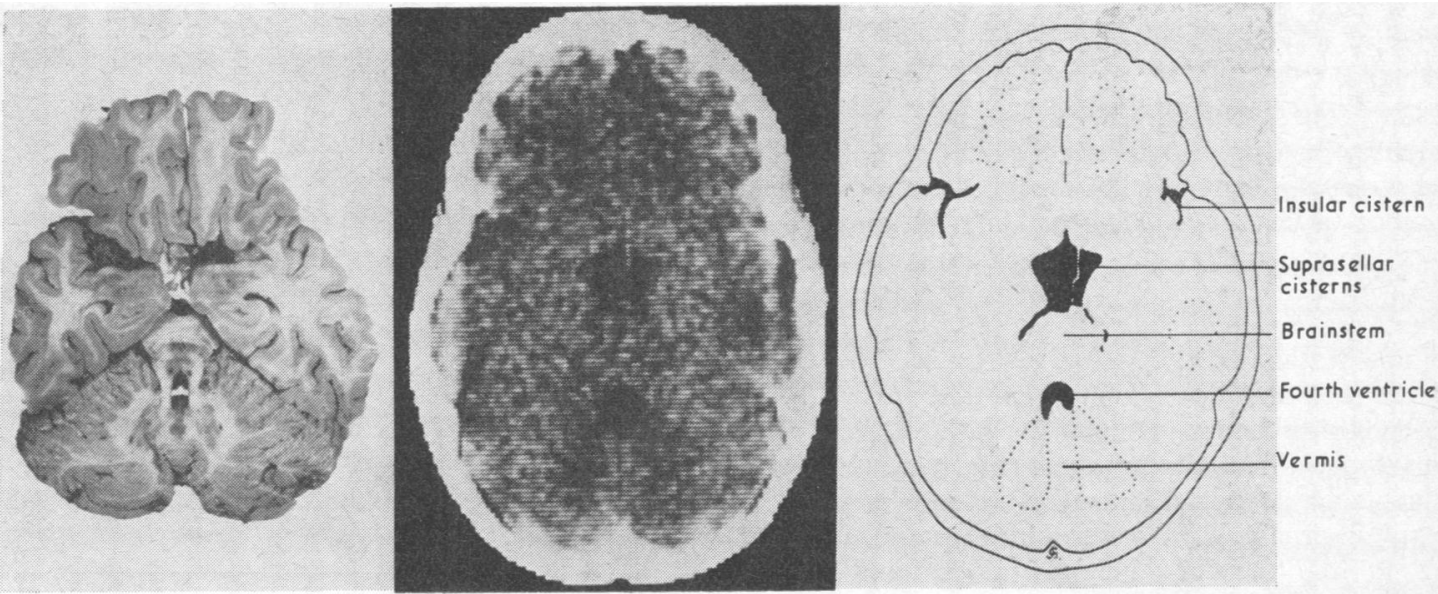

FIG. 4/5
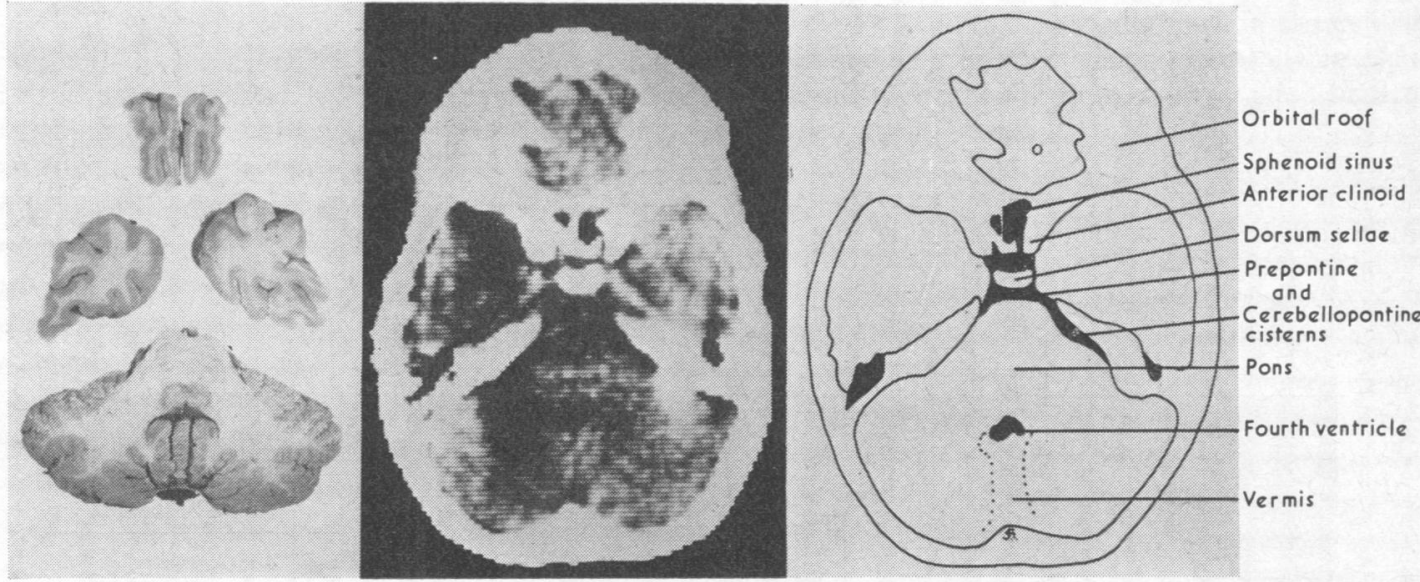

FIG. $4 / 6$

seen in the midline bulging into the posterior segments of the frontal horns. The site of the foramina of Monro is thus marked, for they open into the lateral ventricles posterolateral to the columns of the fornix. Immediately lateral to the frontal horns lie the heads of the caudate nuclei which are generally identifiable as discrete structures. The trigones are well shown at this level, although their margins are not always crisply defined. Often they have a somewhat triangular appearance, with the base laterally and the apex directed anteromedially. The posterior angle of the triangle may continue posteriorly as the occipital horn. The apex of the triangle continues medially towards a group of cerebrospinal fluid filled spaces which lie in the midline at this level. These represent a complex of subarachnoid cisterns and the posterosuperior segment of the third ventricle (Fig. 8). The pineal body lies at the centre of this fluid-containing area, the anatomy of which will be considered in detail later. The thin fluid-containing zone between the trigone and the midline complex of cisterns is the lateral wing of the ambient cistern which runs out into the choroid fissure behind the pulvinar of the thalamus (Fig. 4/3). The lateral ambient wing is separated from the ventricle by the choroid plexus which can often be seen projecting into the trigone. The choroid plexus is frequently identified by the calcium it often contains. Anteromedial to the trigone lies the pulvinar of the thalamus identifiable in its own right. The tail of the caudate nucleus lies at the anterolateral extremity of the trigone but is not seen as a discrete structure. The optic radiation sweeps laterally around the trigone, and is recogni- 
zable as a band of white matter density extending backward from the posterior limb of the internal capsule.

The lowest tomogram incorporating the lateral ventricles usually shows only the inferior segments of the frontal horns (Fig. 4/4). The temporal horns cannot be identified with certainty, although their position can be gauged from related structures. At their predicted site, it is possible to find a vague area where the density is slightly less than the surrounding brain substance. In our experience, clear delineation of the temporal horns implies that they are dilated.

The third ventricle usually lies within the compass of two contiguous tomograms (Fig. 4/3 and 4). On the higher its posterosuperior segment is seen extending anteriorly from the pineal region, where it forms the anterior angle of a diamond-shaped, midline, fluid-containing area surrounding the pineal body. The latter is nearly always identified, even when it contains insufficient calcium to be visible on a conventional radiograph. The posterior angle of the diamond-shaped space represents the cistern of the Great vein of Galen, while the lateral angles are formed by the wings of the ambient cisterns. The third ventricle may be considered as that part of the fluid-containing area anterior to the pineal body. The superior margin of the third

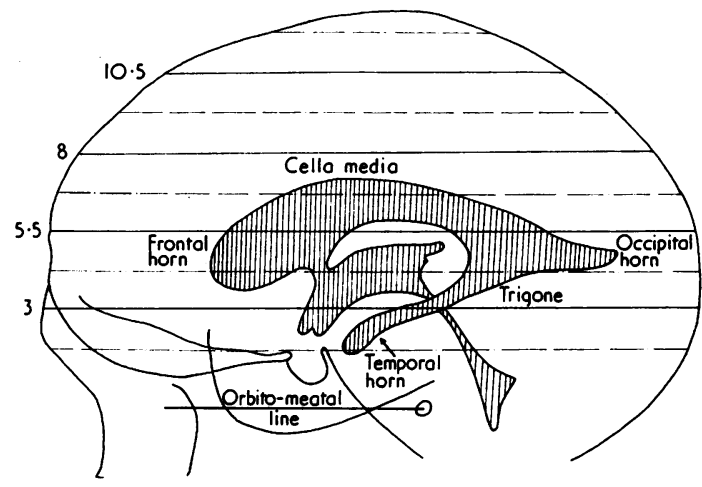

FIG. 5 The ventricular system in relation to transverse axial tomography.

ventricle and its suprapineal recess cannot be defined for they are superimposed on the crura of the fornices anteriorly and the splenium of the corpus callosum posteriorly. What is seen of the posterosuperior part of the third ventricle tapers rapidly as it $\overrightarrow{0}$ extends forward from the pineal region and it mayo disappear anteriorly, being lost by its incorporation $\mathbb{D}$ with the massa intermedia. The anterosuperior $\frac{\text { Pे }}{3}$ 의

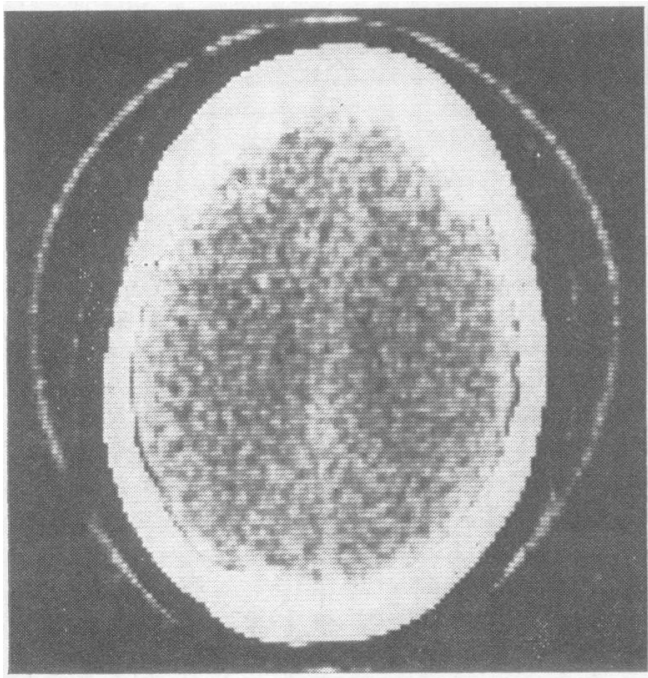

(a)

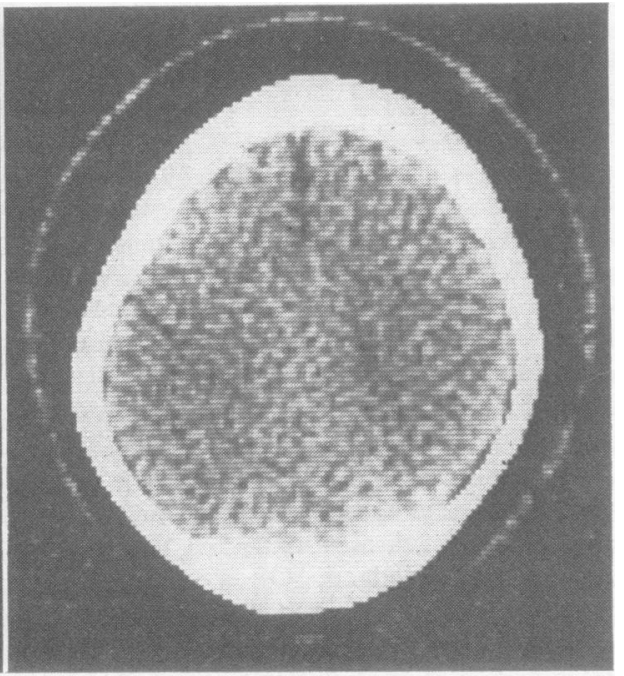

(b)

FIG. 6 (a) The tomogram has grazed the superior aspects of the lateral ventricles which can only just be identified. (b) Slight obliquity of the head causing only the superior aspect of one lateral ventricle (the right) to lie within the tomogram. 


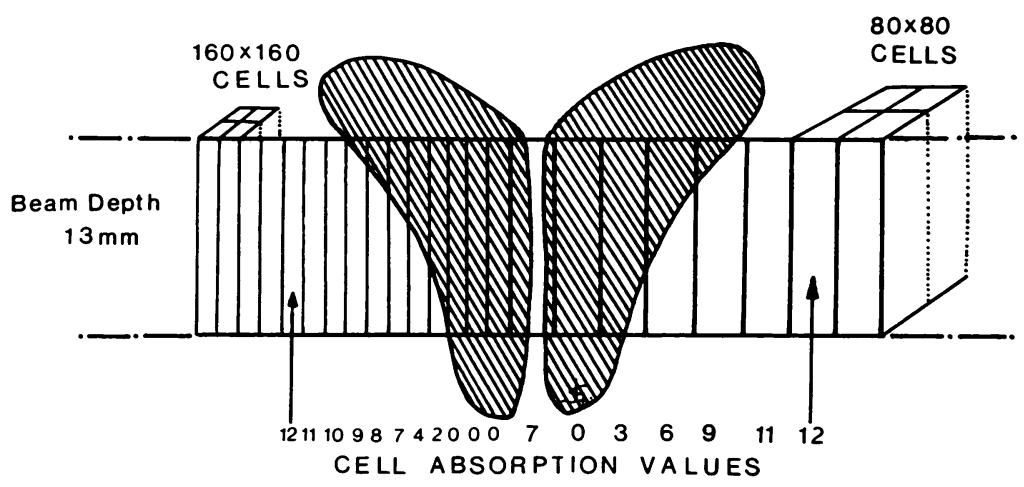

FIG. 7 Because each tomogram is $13 \mathrm{~mm}$ deep the ventricular margin appears as a numerical gradient between cells containing only cerebrospinal fluid and those containing only brain tissue.

segment of the third ventricle may be apparent immediately behind the anterior pillars of the fornices which, as noted previously, allow the foramina of Monro to be located. The thalami, identifiable by their density, form the lateral relations of the third ventricle throughout its anteroposterior course. The lower tomogram containing the third ventricle usually shows the anteroposterior extent more clearly, from the columns of the fornices anteriorly back to the upper midbrain posteriorly where it becomes continuous with the aqueduct. This lower tomogram may also contain the suprasellar cisterns and the superimposition of cerebrospinal fluidcontaining spaces prevents clear definition of the anteroinferior recesses of the third ventricle. The aqueduct, because of its small diameter and oblique course in the plane of the tomogram, cannot be resolve clearly. A slight diminution in density can sometimes be measured in the midbrain at a site which would correspond with the aqueduct, but in practice this structure must be regarded as invisible on the scan unless it is enlarged.

The normal fourth ventricle can always be seen on the appropriate tomograms (Fig. 4/5 and 6) but its shape varies slightly with different angulation of the head in the plane of the tomogram. When the tomogram series lie parallel to the orbitomeatal line, the lowest of the stack usually incorporates the upper segment of the fourth ventricle and it appears as an oval area of low density at the centre of the posterior fossa. The bony orbital roof is likely to be included on a tomogram at this angle and can cause artefactual streaking on the scan which impairs resolution of the posterior fossa. For clear display of the fourth ventricle, it is necessary to flex the head by 10 to 20 degrees with respect to the orbitomeatal line. This projects the posterior fossa upward into the tomogram while the orbital roof is projected below it. A pair of scans taken with this angulation will display the entire fourth ventricle, its lower segment on the

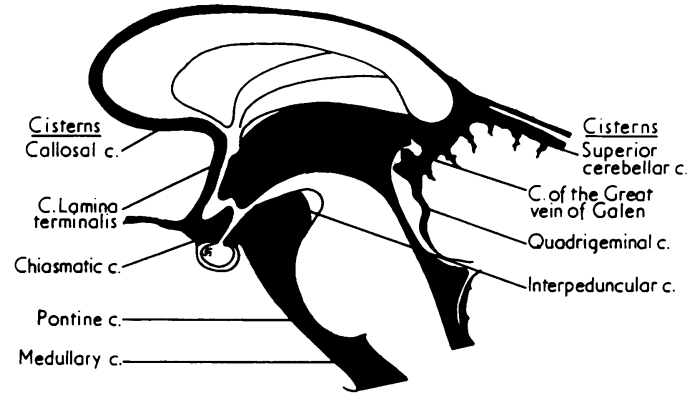

FIG. 8 Diagram to illustrate the relationship of subarachnoid cisterns to the third ventricle.

inferior and its upper segment on the superior tomogram. This head position may also make the posterosuperior recesses of the ventricle more obvious and so changes its contour on the scan (Fig. 9). An outline of the pons is obtained from the pontine cistern anteriorly, the cerebellopontine angle cisterns laterally, and the fourth ventricle posteriorly. The middle cerebellar peduncles turn back from the pons to flank the lateral aspects of the fourth ventricle and the vermis can be resolved as a grey matter band extending in the midline from the posterior limit of the ventricle towards the occipital bone.

SUBARACHNOID CISTERNS Cisterns are visible because they contain fluid but their margins may be lost if they are superimposed on a segment of the ventricular system. Cisterns adjacent to the skull base will not be visible if a tomogram includes the bone beneath them. A thin low density line, one cell deep, can often be recognized on the scan immediately beneath the skull vault. This rim within the skull is a by-product of the mathematical method used to reconstruct the image and does not represent fluid in the sub- 

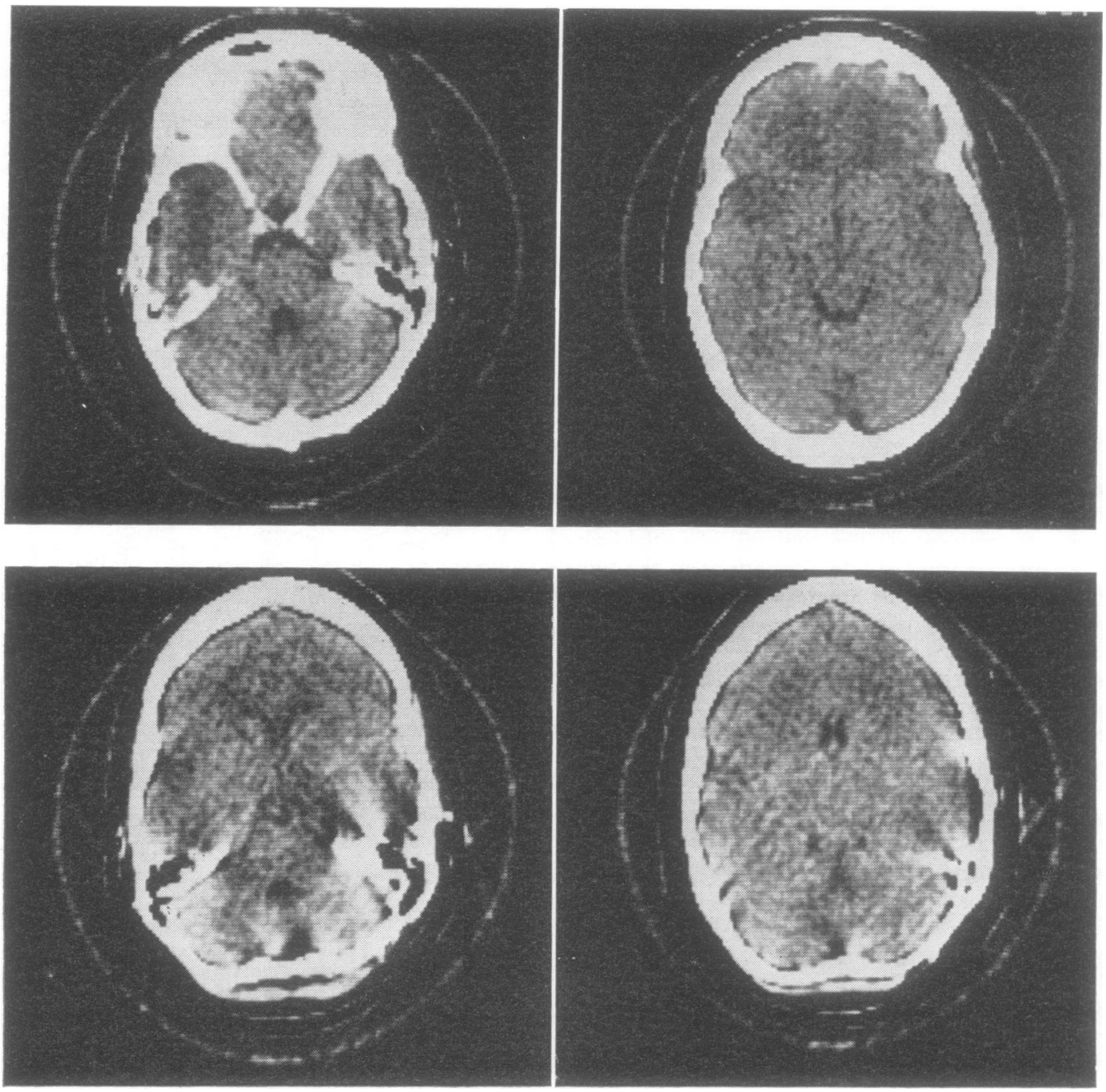

FIG. 9 Scan pair $6 A / B$ (top) are parallel to the orbitomeatal line and the fourth ventricle is seen only on the lower tomogram. Scan pair $9 A / B$ (bottom) are taken with the head flexed $15^{\circ}$ with respect to the orbitomeatal line and the fourth ventricle is now visible on both tomograms. The appearance of the fourth ventricle differs slightly with these two projections.

arachnoid space over the cerebral hemispheres. The subarachnoid spaces cannot normally be defined over the convexity on an 80 by 80 scan, but on the 160 by 160 matrix image cortical sulci can sometimes be seen on the higher tomograms as fine low density linear markings extending inward from the surface. (Fig. 4/1). Deepening of the subarachnoid space or widening of the sulci is readily apparent when cerebral atrophy is present and atrophy also improves the definition of the subarachnoid cisterns.

The cisterna magna can be seen only when its vertical extent is sufficient for the superior segment to be incorporated into a tomogram passing clear of the skull base. The lower segment of the cistern and the vallecula cannot be shown, for they lie too close to the bony floor of the posterior fossa in the region 
of the foramen magnum. The superior segment of the cistern, usually seen only when the head is examined in the flexed position, appears as a midline area of fluid density immediately subjacent to the skull posteriorly. Its depth is generally greatest in the midline where the cerebellar vermis lies anterior to it. Laterally, the cistern tapers as it caps the posterior aspects of the cerebellar hemispheres and its lateral extent is often different on the two sides. The medullary cistern and its lateral recesses are too closely related to the skull base to be shown but the pontine and cerebellopontine-angle cisterns can be seen. The pontine cistern appears as a narrow zone of fluid density immediately behind the clivus and it extends laterally and posteriorly on each side to encircle, and so demarcate, the anterior border of the pons. The cisterns continue further laterally on each side, behind the inner ends of the petrous ridges, to become the cerebellopontine-angle cisterns (Fig. 4/6). The apparent depth of these cisterns and the clarity with which they are shown depends in part upon the angulation of the head. When the clivus is almost vertical to the plane of the tomogram the cisterns are well shown (those cells which lie behind the clivus and petrous ridges will contain chiefly cerebrospinal fluid). When the clivus has a more parallel course with respect to the beam, the cisterns are likely to be obscured by superimposition of the clivus anteriorly and the brain stem posteriorly. Any head movement during scanning is likely to cause artefactual streaking backward from the clivus and petrous bones into the posterior fossa so masking these cisterns. Even without head movement, an artefactual low density streak often extends between the tips of the petrous ridges and obscures the anterior border of the pons. The prepontine cistern is continuous anterosuperiorly with the interpeduncular and crural cisterns. When sharply delineated, these cisterns outline the anterior border of the midbrain and display the cerebral peduncles. Anteriorly, the cisterns encircling the anterior midbrain are continuous with the chiasmatic cistern, which appears as a median area of fluid density above the sella (Fig. 4/5). The anterior clinoid processes, tuberculum, and dorsum sellae are often projected into this low density zone from below, while the anteroinferior segment of the third ventricle is projected from above. The anterior limit of the suprasellar cisterns may give rise to a midline band of fluid density representing the interhemispheric fissure between the frontal lobes, while its anterolateral corners continue into the lateral fissures as they extend forward and laterally towards the pteryon. It is possible to identify the circular (insular) cisterns on some normal scans as semilunar zones of fluid density, concave medially, which lie parallel and deep to the surface of the hemisphere overlying the island of Reil (Fig. 4/4). Widening of the lateral and interhemispheric fissures together with prominence of the insular cisterns occurs in cerebral atrophy.

The posterior aspect of the midbrain is capped by the quadrigeminal cistern, which can be regularly identified on both the 80 by 80 and 160 by 160 matrix scans (Fig. 4/4). It provides a landmark on the scans defining not only the midbrain but also the level of the tentorial hiatus. The lateral extremities of the quadrigeminal cistern extend anterolaterally around the midbrain to become continuous with the crural cisterns. The lateral conduits are termed the ambient cisterns and it is often possible to demonstrate the entire midbrain from its encircling cisterns. The quadrigeminal cistern continues upward over the superior colliculus into the cistern of the great vein of Galen, which, as was noted previously lies immediately behind the posterosuperior aspect of the third ventricle (Fig. 4/3). The lateral extremities of the Galenic cistern are prolonged behind the pulvinar of the thalamus as the lateral wings of the ambient cistern. Posteriorly, the Galenic cistern is continuous with the subarachnoid space beneath the tentorium and overlying the superior vermis. This is the superior cerebellar cistern. On a normal scan, it is possible to detect fine semilunar zones of reduced density radiating anterolaterally into each cerebellar hemisphere from the superior cerebellar cistern. These linear areas represent fluid present in the spaces between cerebellar folia. While the superior cerebellar cistern is variable in size, widening of the spaces between the folia indicates cerebellar atrophy.

CEREBRAL HEMISPHERES Because computerized tomography can detect the slight measurable difference in $x$-ray absorption between grey (absorption value 18-30) and white matter (11-18), certain cerebral structures may be identified in their own right. The density difference between grey and white matter almost certainly depends upon several factors. First, grey matter is the more vascular and the density of unclotted blood is relatively high (absorption values 13-31; Scott et al. (1974). Second, the accumulation of high atomic number substances, like iron, will affect the grey matter preferentially and third, white matter contains more lipid material (myelin sheaths) than grey.

Further definition of certain cerebral structures can be gained from the outline provided by the ventricular system or subarachnoid cisterns.

The cortical grey matter mantle is recognizable over the surface of the cerebral hemispheres not only subjacent to the skull but also as a midline band in relation to the interhemispheric fissure (Fig. 4/1). 

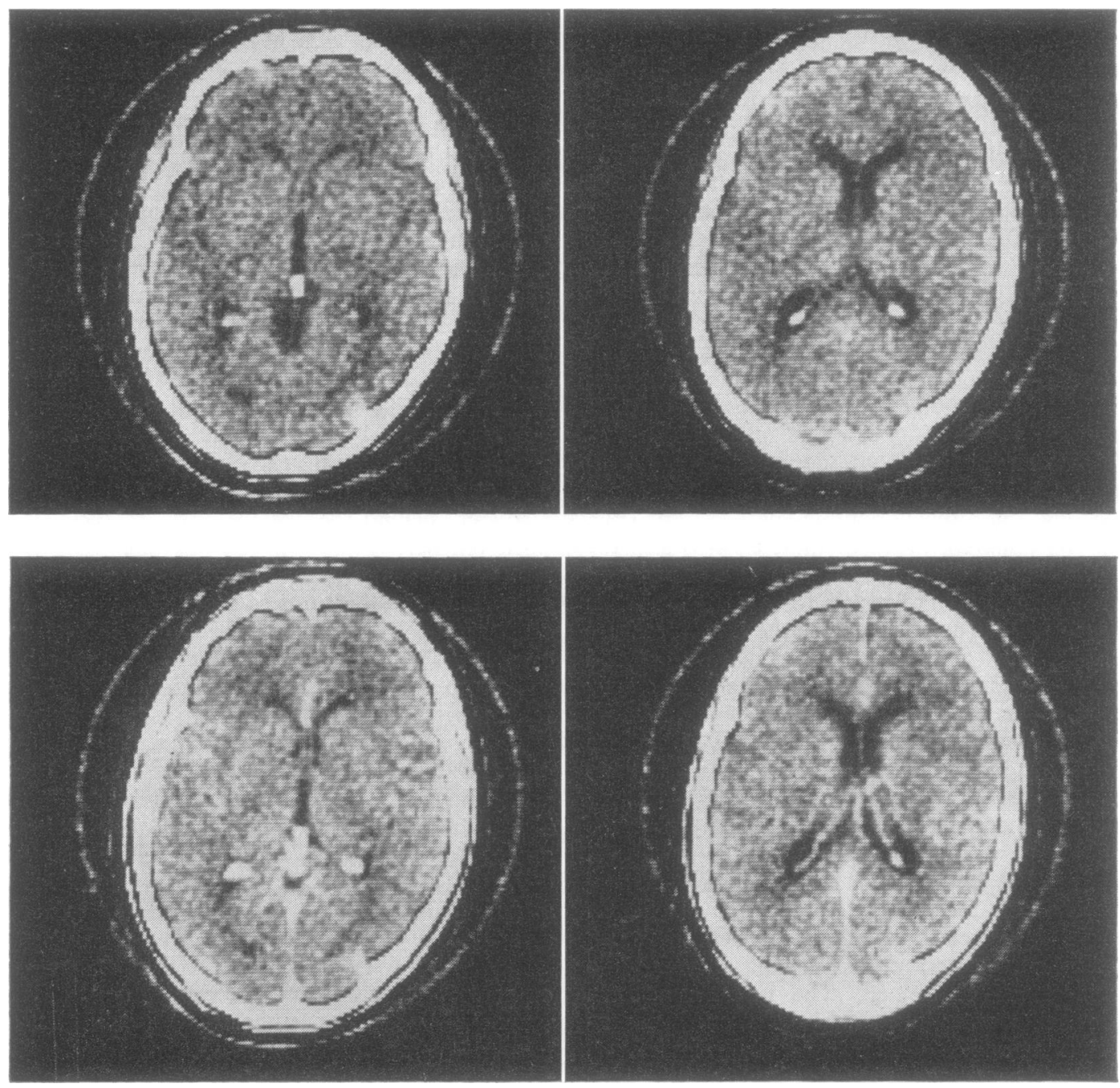

FIG. 10 Scan pairs $2 A / B$ (top) before, $3 A / B$ (bottom) after the injection of $100 \mathrm{ml}$ sodium iothalamate (Conray 420) intravenously, to demonstrate normal enhancement of tissue density.

The distinction between cortical mantle and underlying white matter is least obvious in infants and the density difference tends to become more obvious with advancing age. These changes may reflect the increasing degree of myelination in infancy and the accumulation of high atomic number substances with ageing. Further, the total accumulation of calcium in the vasculature might be expected to affect the grey matter more obviously. Conversely with increasing age, the enlargement of those sulci which are not defined as discrete structures might be expected to lower the density of the cortical mantle by their contained cerebrospinal fluid. On tomograms incorporating the insula, it is possible to recognize a vague area of grey matter density extending inward from the cortical mantle to lie along the margins of the circular cistern.

The heads of the caudate nuclei are regularly seen as oval zones of grey matter density immediately lateral to the frontal horns (Fig. 4/3). Asymmetry of $N$ these nuclei is commonly seen and presumably represents their differential incorporation; a result of 
slight obliquity of the tomogram. The thalami are recognizable in the majority of patients, their medial margins can be gauged from the third ventricle for they extend along it from the foramina of Monro to its posterior extremity. The posterior aspect of the pulvinar is outlined by the trigone and wing of the ambient cistern. The lateral thalamic margins are defined by the posterior limbs of the internal capsules.

The putamen and globus pallidus (lentiform nuclei) are not as well defined as the foregoing deep nuclei and they merge laterally with the grey matter of the insula. It is always possible to locate the internal capsule as a band of white matter density lying between the head of the caudate and lentiform nucleus anteriorly and the thalamus and lentiform nucleus posteriorly. The posterior limb is generally rather better demarcated than the anterior. The posterior limb of the internal capsule is prolonged backward as the optic radiation, while the anterior limb continues forward as the frontal radiation. The general course of both these large white tracts can be discerned. Oedema associated with cerebral tumours or abscesses tends to follow white matter pathways and widening of the internal capsule can sometimes be seen. The external capsule, which is not normally visible, may be highlighted in this way.

Scans taken after the intravenous injection of an iodine-containing contrast medium (Fig. 10) enable the larger intracranial blood vessels to be seen. The anterior cerebral arteries cause enhancement in tissue density at the posterior end of the interhemispheric fissure anterior to the septum pellucidum. The middle cerebral artery trunks may be identified in the lateral fissures and their branches cause enhancement in the insular cistern. Occasionally, the posterior cerebral arteries are seen as ring-like, enhanced zones around the brain stem. The choroid plexus of the third and lateral ventricles may be identified by their enhancement following intravenous contrast, and enhancement along the interhemispheric fissure above the lateral ventricles reflects the vessels in relation to the falx. Constant enhancement of the great vein of Galen, inferior sagittal, and straight sinuses allows these structures to be defined. Some overall increase in tissue density in the cortical grey mantle, basal ganglia, and thalamus follows large intravenous iodine injections and may allow clearer delineation of these structures.

CEREBELLUM AND BRAIN-STEM It is possible to define within each cerebellar hemisphere a zone of white matter density which corresponds with the corpus medullare and the cerebellar cortex and vermis are detected by their higher density (Fig. 4/5). The site of the middle cerebellar penduncles is given by the pontine, cerebellopontine angle cisterns, and the fourth ventricle. The brain-stem itself is delineated by its encircling subarachnoid cisterns and the midbrain is often sufficiently well encompassed to show the cerebral peduncles and collicular plate. The upper pons is similarly displayed by its anterior cisterns but the lower pons and medulla are generally obscured by their proximity to the skull base. None of the brain-stem nuclei can be seen in their own right but the position of the larger nuclei may be

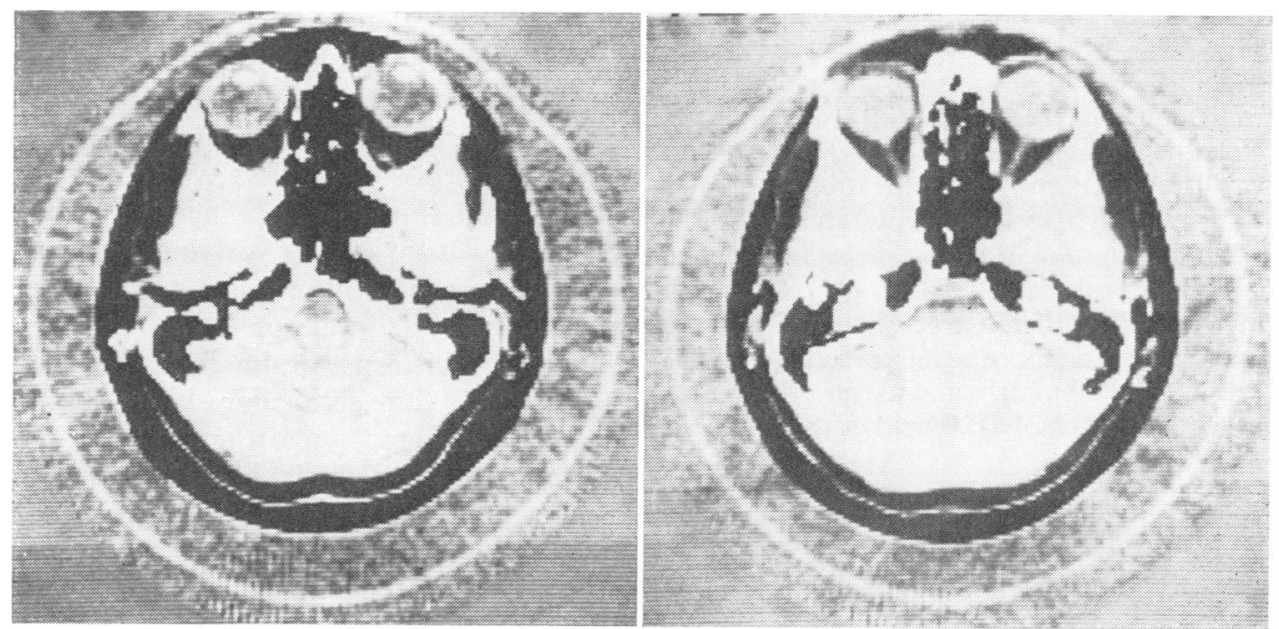

FIG. 11 Normal EMI Scan of orbits. 
anticipated from the brain-stem contour at a given level.

\section{Orbital compartment}

(Fig. 11)

With careful positioning of the head, it is possible to obtain a pair of contiguous tomograms each $8 \mathrm{~mm}$ deep, through the orbits which pass clear of their superior and inferior bony margins. It is important to avoid incorporating either the roof or floor of the orbit into these tomograms, since their bony density will obscure adjacent structures. Seen in transverse section, the orbits lie on either side of the nasal and paranasal cavities. The bony medial and lateral orbital walls mark out a triangular space with the globe anteriorly at its base. The retrobulbar space is filled chiefly by fat whose very low absorption values $(-10$ to -70$)$ provide a natural contrast against which the optic nerves and some extraocular muscles are contrasted.

The globe, seen in transverse section, appears as a ring whose absorption values fall between 5 and 25 surrounding the less dense vitreous whose values are between -15 and +5 . The high peripheral density is produced by the sclera and its attached musculature. Within the ring, on the 160 by 160 matrix, the lens can be identified anteriorly, for it is more dense than the vitreous. When the tomogram incorporates either the inferior or superior pole of the globe, rather than passing through its equator, the ring-like appearance is replaced by a sphere of even density. Any obliquity of the head will result in the beam passing through the eyes at different levels and this may result in one eye appearing larger than the other. The apparently larger eye will project further forward and an erroneous impression of proptosis can be given.

The optic nerve can always be seen extending forward and laterally from the orbital apex to the globe. While the optic nerve is always identifiable on the 80 by 80 matrix scan, its delineation is greatly improved by the high resolution system. With the 160 by 160 matrix system, it is also possible to define the medial and lateral rectus muscles in the majority of patients and the other extraocular muscles are occasionally seen. The extraocular muscles are not shown by the 80 by 80 matrix and as yet other intraocular nerves are not resolved by either matrix.

\section{DISCUSSION}

Computerized tomography produces an image which cannot be thought of in quite the same way as a radiograph. The result is a mathematical reconstruction of a 'slice' of the patient's head in terms of its density, and it is therefore possible to have areas of abnormal density on the image which are not due to abnormal tissue but to some on fault in the mathematical reconstruction. Such errors may occur when the patient's head moves during scanning, when certain faults in the apparatus occur, and also when the tomogram includes marked density changes sequentially throughout the 'slice'. The latter situation obtains in the region of the skull base when a $\stackrel{5}{\stackrel{5}{9}}$ sequence of bone and brain are present. The abnormal areas that result can nearly always be recognized as artefacts and with experience they rarely lead to diagnostic confusion.

The anatomy of the brain as seen in the trans- कs verse plane is seldom considered in the inter- $\vec{\circ}$ pretation of conventional radiographs, neurolo- $\overrightarrow{\vec{\omega}}$ gists, neurosurgeons, and radiologists being $\stackrel{\omega}{\rho}$ more familiar with lateral and anteroposterior $\bar{\Xi}$ projections. Because a vertical series of trans-? verse tomograms is achieved by computerized $\stackrel{\omega}{\omega}$ tomography, it is possible to gain a three $\overrightarrow{0}$ dimensional representation of the intracrania $c$ and orbital compartments. It must be stressed again, however, that each tomogram represen $\frac{8}{8}$ the superimposition of all those structures cor tained within a 'slice' of the head either 13 of $8 \mathrm{~mm}$ in thickness. As a result of norm通 anatomical variation, tomograms at a given leve् in different patients may have a dissimil appearance and it is wrong to think in terms of standardized slices. Changing the vertical plane of a tomogram in one patient by only a few millimetres can strikingly change its appearance, and altering the angulation of the head may have the same effect. These considerations are perhaps most important when interpreting scans taken through the posterior fossa. When the tomogram 'grazes' the skull base, the incorporation of bone effectively masks the overlying soft tissues, an essential consideration when trying to delineate the inferior frontal or temporal lobes, the suprasellar cisterns, brain stem, and the inferior 3 aspects of the cerebellar hemispheres. It is important to recognize that tomograms at the appropriate level will include structures on either $\mathrm{O}$ side of the tentorium, and this occasionally gives rise to difficulties in siting lesions above or below it. Any lateral obliquity of the head may cause $N$ different structures from the right and left hemicrania to be incorporated in the tomogram, $N$ 
and so lead to a confusing asymmetry in its appearance.

The 160 by 160 matrix scans undoubtedly give an image of far greater definition than the 80 by 80 matrix allows. This, together with the $225^{\circ}$ scan providing 54000 transmission readings, has proved especially valuable in delineating posterior fossa and orbital structures. When outlining the periphery of a structure, not only must the cell size in the transverse plane be considered, but also the depth of the cells. Thus, numerical gradients in density occur at the edges of definable structures which pass obliquely through the tomogram, and this is significant when estimating dimensions.

Computerized tomography is perhaps the most significant advance in the use of $x$-rays for diagnosis since their inception. Certainly the technique is already revolutionizing the investigative routine of patients suspected of structural intracranial or orbital disease. It also promises to increase our understanding of many pathological processes; their natural history and response to treatment, without exposing patients to hazard. Images of the brain can now be obtained during life, without anaesthetizing the subject and without introducing contrast media such as air which may distort the findings. A new chapter in neurology has clearly been opened.

We should like to thank Dr W. G. P. Mair for his help in preparing the brain slices, Miss V. Fullom for her radiographic assistance, $\mathrm{Mr} \mathrm{A}$. $\mathrm{H}$. Prentice and $\mathrm{Mr} \mathrm{J}$. Mills for photography and Miss S. Andrews for her secretarial aid.

\section{REFERENCES}

Hounsfield, G. N. (1973). Computerized transverse axial scanning (tomography). Part I. Description of the system. British Journal of Radiology, 46, 1016-1022.

Scott, W. R., New, P. F. J., Davis, K. R., and Schnur, J. A. (1974). Computerized axial tomography of intracerebral and intraventricular haemorrhage. Radiology, 112, 73-80. 